\title{
Renal artery embolization following aortobifemoral bypass: A case report
}

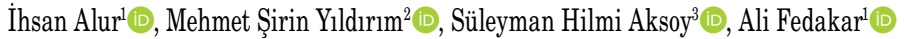 \\ ${ }^{1}$ Department of Cardiovascular Surgery, Hisar Hospital Intercontinental, Istanbul, Turkey \\ ${ }^{2}$ Department of Anesthesiology and Reanimation, Hisar Hospital Intercontinental, Istanbul, Turkey \\ ${ }^{3}$ Department of Radiology, Hisar Hospital Intercontinental, Istanbul, Turkey
}

\begin{abstract}
Renal artery occlusion and infarction following aortobifemoral bypass is extremely rare complication and its diagnosis is challenging. Early diagnosis and treatment of this pathology is critical. Herein, we present a 49-year-old male case who was operated for peripheral artery disease and in whom subtotal renal infarction developed in the postoperative fifth day.
\end{abstract}

Keywords: Aortobifemoral, bypass, embolization, renal artery.

Renal artery occlusion and renal infarction following aortobifemoral bypass is extremely rare complication and are difficult to diagnose. Risk factors include atherosclerosis, renal parenchymal diseases such as nephrotic syndrome and glomerulonephritis, renal artery aneurysms and dissection, dissecting aortic aneurysms, endocarditis-related septic embolization, systemic lupus erythematosus, sickle-cell anemia, and fibromuscular dysplasia of the renal arteries. ${ }^{[1]}$ Renal infarction may completely asymptomatic or may present with typical signs and symptoms such as pyrexia, nausea/vomiting, and flank pain, or loin tenderness. ${ }^{[1,2]}$

Herein, we present a case operated for peripheral artery disease in whom subtotal renal infarction developed in the postoperative fifth day in the light of literature data.

\section{CASE REPORT}

A 49-year-old male case was admitted with the complaint of claudication. Physical examination revealed a pulse rate of $84 / \mathrm{min}$ and a blood pressure of $120 / 90 \mathrm{mmHg}$ with normal other system findings. Right femoropopliteal pulses were palpable, right dorsalis pedis (DP), and tibialis posterior (TP) pulses were non-palpable, while the left femoral, popliteal, DP, and TP pulses were non-palpable. He had left ischemic non-healing wound. Coronary angiography demonstrated normal left main coronary artery, plaque formation in the first diagonal (D1) branch of the left anterior descending artery (LAD), 70\% stenosis in the D1, normal circumflex $(\mathrm{Cx})$ artery, $40 \%$ stenosis in the proximal of the right coronary artery, and $60 \%$ stenosis in the mid-segment. As the patient had no anginal complaint, medical follow-up was recommended. Peripheral angiography showed 100\% occlusion in the right main iliac artery with distal filling with collaterals, while there was $95 \%$ occlusion in the left main iliac artery and 100\% occlusion in the left superficial artery with distal filling with collaterals. He suffered from intermittent claudication which started after a 50 -meter walk. ${ }^{[2]}$ Based on these findings, the patient was scheduled for surgery and a written informed consent was obtained.

The abdomen and peritoneum were explored through a left median laparotomy under general anesthesia. The intestines were carefully pulled out and the retroperitoneum was reached. The abdominal aorta and both main iliac arteries were released.

Received: December 14, 2018 Accepted: February 11, 2019 Published online: March 08, 2019

Correspondence: İhsan Alur, MD. Hisar Hastanesi Intercontinental Kalp ve Damar Cerrahisi Bölümü, 34768 Ümraniye, İstanbul, Turkey. e-mail: alur_i@hotmail.com 
The common femoral artery and superficial femoral artery were released through a right and left femoral incision and the profunda femoral arteries were released by encircling with a vessel tape. Following heparinization, longitudinal aortotomy was performed through proximal and distal cross-clamping of the abdominal aorta. An aortic end-to-side anastomosis was made using a Dacron ${ }^{\circledR}$ graft $(16 / 8 \mathrm{~mm})$. The graft extensions were placed into both femoral arteries through a retroperitoneal tunnel and aortobifemoral bypass grafting was performed. A soft drain was inserted into the peritoneum and all layers were closed in a standard fashion. The popliteal artery was, then, prepared and left femoropopliteal bypass using crossclamping was applied. A soft drain was inserted into the left popliteal region. The operation was completed after bleeding control.

Prophylactic antibiotherapy was initiated with cefazolin $1 \mathrm{~g}$ daily ( $\mathrm{q} 6 \mathrm{~h}$, intravenous). The drains were removed in the postoperative first day. Positive bowel sounds were noted with gas/stool output. Oral nutrition was initiated. In the postoperative third day, the patient suffered from fever $\left(38.5^{\circ} \mathrm{C}\right)$, nausea/vomiting, and flank pain. Blood and urine culture tests were performed. Complete blood count test results were as follows: white blood cell (WBC): $14.61010^{3} / \mu \mathrm{L}\left(4.16-10.210^{3} / \mu \mathrm{L}\right)$ and C-reactive protein: $23.53 \mathrm{mg} / \mathrm{dL}(0-0.5 \mathrm{mg} / \mathrm{dL})$. Biochemistry test results were as follows: aspartate aminotransferase (AST): 63 IU/L (0-37 IU/L),
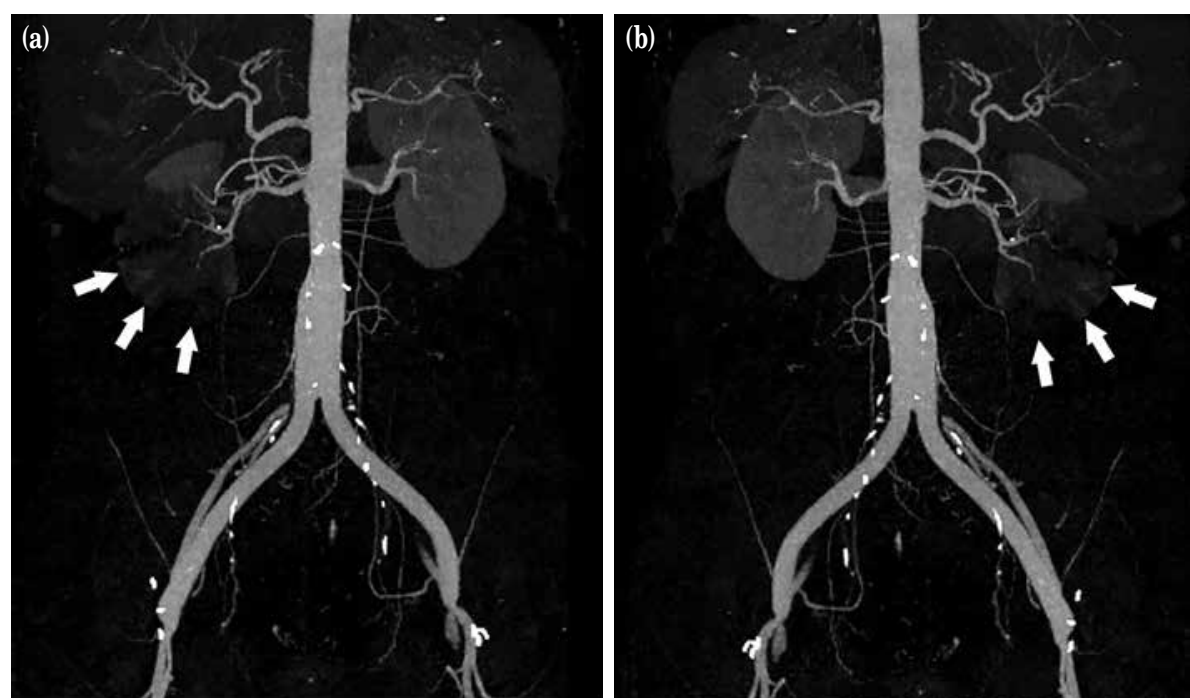

(c)
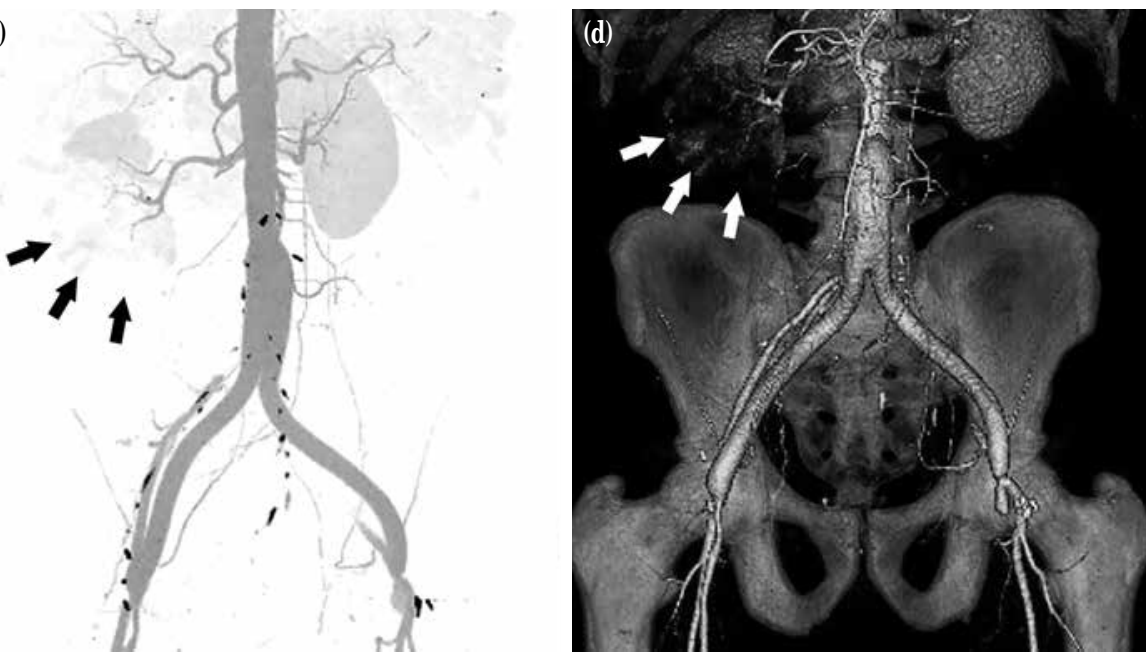

Figure 1. (a) Anterior view of abdominal computed tomography showing right subtotal renal infarction (white arrows); (b) Posterior view of abdominal computed tomography showing right subtotal renal infarction (white arrows); (c) Anterior view of abdominal computed tomography showing right subtotal renal infarction (black arrows).

(d) Colored and three-dimensional image of right subtotal renal infarction. 
alanine aminotransferase (ALT): 115 IU/L (0-41 IU/L), lactate dehydrogenase (LDH): $886 \mathrm{U} / \mathrm{L}$ (135-225 U/L), urea: $58.8 \mathrm{mg} / \mathrm{dL}$ (11-61 mg/dL), and creatinine: $1.53 \mathrm{mg} / \mathrm{dL}(0.67-1.27 \mathrm{mg} / \mathrm{dL})$. Abdominal examination showed hypoactive bowel sounds with lost gas/stool output. A plain abdominal X-ray in the standing position revealed multiple air-fluid levels. The patient was consulted with a general surgeon and diagnosed with subileus. Oral nutrition was discontinued, and parenteral fluid therapy was initiated. Abdominal contrast-enhanced computed tomography (CT) showed a subtotal infarction of the right kidney (Figure 1). Aortobifemoral Y-graft was patent without any intraabdominal pathology. Two days later, bowel sounds were found to be normoactive and gas/stool output started. No fever or flank pain was seen. On Day 10, the WBC and elevated parameters returned to normal. There was no reproduction in the blood and urine culture. On Day 12 postoperatively, the patient was uneventfully discharged.

\section{DISCUSSION}

Fever of unknown origin, flank pain, nausea/ vomiting, increased bowel sounds, and lost gas/stool output may indicate ileus, intraabdominal infection, abscess, or foreign bodies in patients undergoing aortobifemoral bypass. In the differential diagnosis, renal infarction is also considered. In our case, renal infarction was evident. Previous studies have suggested that renal artery occlusion or renal infarction should be considered in patients with fever of unknown origin, flank pain and hematuria with any pathology leading to embolization such as atrial fibrillation, endocarditis, or atherosclerotic aorta. ${ }^{[1,3]}$ In our case, however, no atrial fibrillation or endocarditis was present. In addition, no gross hematuria was seen. However, we were unable to detect microscopic hematuria due to the lack of samples for complete urinalysis.

Renal infarction is usually seen in elderly patients with a mean age of 67 years. ${ }^{[4]}$ It affects both sexes and both kidneys. ${ }^{[4]}$ In contrast to the literature data, our case was relatively young. In addition, nearly half of patients with renal infarction experience fever and sudden systemic arterial hypertension due to activated renin-mediated mechanism. ${ }^{[4-6]}$ We believe that embolization is related to the cross-clamp we put in the proximal aorta and the clamp-induced plaque or thrombus mobilization/shift may occur. Moreover, patients may present with extra-renal systemic embolization manifestations such as mesenteric ischemia, limb ischemia, and cerebral infarction. Our case was completely normotensive without extra-renal systemic embolization. Some of the parameters studied in laboratory tests such as alkaline phosphatase, fibrinogen, CRP, AST, ALT, and WBC can be used as renal infarction biomarkers in clinical practice. In particular, $\mathrm{LDH}$ is known as the most sensitive biomarker. $^{[5,6]}$ In our case, similarly, some of these parameters were found to be elevated and returned to normal after recovery. Furthermore, contrastenhanced CT is highly effective in the diagnosis of renal infarction with $85 \%$ sensitivity. ${ }^{[6]}$ In our case, CT yielded the definite diagnosis.

In clinical practice, renal infarction is usually misdiagnosed as acute pyelonephritis, lymphoma, or metastatic cancer. Timely diagnosis is, therefore, of utmost importance to initiate thrombolytic and anticoagulant therapies or to perform embolectomy, minimizing renal function loss. ${ }^{[5]}$ Standard treatment is anticoagulation with or without thrombolytics. ${ }^{[1]}$ In many patients with acute renal infarction, renal insufficiency at varying degrees may be seen. The degree of renal insufficiency depends on the unilateral or bilateral renal involvement and the progression rate. No renal insufficiency was seen in our patient; only mild elevation in the creatinine levels was observed which returned to normal within five days.

In conclusion, cardiovascular surgeons should consider that such a complication may develop during aortic surgery and that they should experience the management of this problem. In this report, we highlight the importance of early recognition of this complication.

\section{Declaration of conflicting interests}

The authors declared no conflicts of interest with respect to the authorship and/or publication of this article.

\section{Funding}

The authors received no financial support for the research and/or authorship of this article.

\section{REFERENCES}

1. Bouassida K, Hmida W, Zairi A, Hidoussi A, Jaidane M, Slama A, et al. Bilateral renal infarction following atrial fibrillation and thromboembolism and presenting as acute abdominal pain: a case report. J Med Case Rep 2012;6:153.

2. Yildızhan İ, Mert B, Özkaynak B, Gülben Kük Z, Polat A. Directional atherectomy in femoropopliteal occlusive diseases: Our midterm results. Turk J Vasc Surg 2019;28:1-8.

3. Bande D, Abbara S, Kalva SP. Acute renal infarction secondary to calcific embolus from mitral annular calcification. Cardiovasc Intervent Radiol 2011;34:647-9. 
4. Lopez VM, Glauser J. A case of renal artery thrombosis with renal infarction. J Emerg Trauma Shock 2010;3:302.

5. Napoli A. An unusual presentation of renal infarction. Am J Emerg Med 2004;22:437-8.
6. Antopolsky M, Simanovsky N, Stalnikowicz R, Salameh $\mathrm{S}$, Hiller $\mathrm{N}$. Renal infarction in the ED: 10-year experience and review of the literature. Am J Emerg Med 2012;30:1055-60. 